\title{
Performance of Longus Colli Muscle in Women with and without Forward Head Posture
}

\author{
F. Bokaee ${ }^{1}$, F. Dehghan Manshadi² \\ ${ }^{1}$ Department of Physiotherapy, Faculty of Rehabilitation Sciences, Isfahan University of Medical Sciences, Isfahan, \\ Iran \\ 2 Department of Physiotherapy, Faculty of Rehabilitation Sciences, Shahid Beheshti University of Medical \\ Sciences, Tehran, Iran
}

\author{
CORRESPONDING AUTHOR: \\ Fateme Bokaee \\ Department of Physiotherapy \\ Faculty of Rehabilitation Sciences \\ Isfahan University of Medical Sciences \\ Hezar-Jerib Street \\ Isfahan, Iran \\ E-mail: fatemebokaee@yahoo.com
}

DOI:

10.32098/mltj.01.2021.20

LEVEL OF EVIDENCE: $3 B$

\begin{abstract}
SUMMARY
Background. Forward head posture (FHP) is the most common poor postures in patients with shoulder and neck pain. Longus colli (LCo) muscle has important role in maintaining normal posture of the cervical spine and dysfunction of this muscle may lead to abnormal posture of the cervical spine. Ultrasonography is one of the most common methods used to evaluate muscle performance. The aim of this study was to compare performance of LCo muscle between women with and without FHP.

Methods. This cross-sectional study included 70 women with and without FHP (35 in each group). Thickness of LCo muscle in resting state, contraction state $(50 \%$ of maximal isometric contraction of craniocervical flexion) and thickness change of this muscle between resting and contraction state were measured by ultrasonography and compared between two groups using Independent-Samples T test.

Results. The results indicated that there was no significant difference between two groups regarding thickness of LCo muscle in resting and contraction state but, thickness change of this muscle between resting and contraction state was significantly smaller in women with FHP compared with control group.

Conclusions. The results of this study indicated that measurement of thickness change of LCo muscle between resting and contraction state seems to be a better parameter than thickness of this muscle in resting or contraction state for assessment of performance of this muscle.
\end{abstract}

KEY WORDS

Craniocervical; forward head posture; Longus colli; ultrasonography; women.

\section{BACKGROUND}

Posture can be defined as the alignment of body segments in a specific time (1). Forward head posture (FHP) is one of the most prevalent abnormal posture (2) and FHP is defined as forward positioning of the head relative to the trunk in sagittal plane (3). Previous studies indicated that FHP is linked with many musculoskeletal dysfunction such as shoulder pain, neck pain, headache and craniofacial pain (4). Abnormal sitting postures (e.g. working with computers at an improper height of computer screen) can lead to FHP (5). The prevalence of this abnormal posture has been increased because of increased time spent working on a computer. Longus colli (LCo) muscle has important role in controlling cervical lordosis and maintaining posture of the cervi- cal spine. This muscle is an important stabilizer to maintain normal head on neck posture (6). Weakness of this muscle decrease proprioception function and ability of this muscle to control posture of the cervical spine (7). Previous studies indicated that FHP is associated with weakness of upper cervical flexor muscles in patients with cervicogenic headache (8).

Evaluation of muscle thickness can indicate muscle function and strength (9). Magnetic Resonance Imaging (MRI), Computed Tomography (CT) scan, and ultrasonography are common methods which are used to measure muscle thickness. Ultrasonography is considered an accessible, cost effective, reliable and noninvasive method for evaluation of muscle thickness and activity $(10,11)$. Previous stud- 
ies indicated that muscle thickness changes is correlated with amplitude of electromyographic signal (11) and more change in muscle thickness is indicative of more muscle activity (11). So quantifying muscle thickness change may indirectly represent muscle function (12).

By regarding high incidence of FHP among computer users (especially in youth) and increased usage of computer, the importance of considering this abnormal posture in researches is highlighted. Although some previous studies conducted on FHP but, to the best of the authors' knowledge, there is lack of studies evaluated thickness change of LCo muscle during craniocervical (CC) flexion in FHP. Keeping these points in mind, the present study was designed to compare thickness of LCo muscle in resting state, contraction state $50 \%$ of maximal isometric contraction of CC flexion) and thickness change of this muscle between resting and contraction state between women with and without FHP. Similar studies can promote knowledge about performance of LCo muscle in FHP and help for better management of FHP.

\section{MATERIALS AND METHODS}

\section{Participants}

Participants of this cross-sectional study were seventy asymptomatic women, aged between 20 and 40 years. This study was a part of a larger study. Sample size in this study was considered 35 in each group (with and without FHP). This sample size was calculated using this formula:

$$
\mathrm{N}=\left(\mathrm{Z}_{1-\alpha / 2}+\mathrm{z}_{1-\beta}\right)^{2}\left(\alpha_{1}^{2}+\alpha_{2}^{2}\right) /\left(\mu_{1}-\mu_{2}\right)^{2}
$$

where:

- $\quad \alpha: 0.05$

- $\beta: 0.1$

- power: $90 \%$;

- $\alpha_{1}: 1.81$

- $\alpha_{2}: 2.34$

- $\mu_{1}: 2.86$

- $\mu_{2}: 4.6(13)$.

Participants were divided into two groups (with and without FHP, 35 in each group) according to the craniovertebral angle (CVA) measured by photographic images. Evaluation of CVA is described later in this article. The inclusion criteria for both groups were women aged 20-40 years. Participants with CVA greater than $48^{\circ}$ were included in the control group and participants with CVA less than $48^{\circ}$ were included in the FHP group (14). In this study our partici- pants were only women subjects to exclude possible effects of gender on the results of this study.

The exclusion criteria for both groups were as follows: neck or shoulder pain, radicular pain of the upper extremity, history of cervical surgery, severe thoracic kyphosis, history of musculoskeletal or neuromuscular diseases, previous or current injuries in neck and shoulder, history of facial trauma or surgery, participation in any sports activities, hearing impairments requiring the use of a hearing aid, recurrent middle ear infections in the previous 5 years, persistent respiratory difficulties over the last 5 years that interfered with daily activities, any visual impairment not corrected by glasses, central nervous system disorders, and temporomandibular disorders (3).

\section{Evaluation of CVA}

At first, the aims and procedures of the current study were explained to the participants. Prior to data collection, participants were asked to sign informed consent form. The present study meets the ethical standards of the journal. The study had ethical approval from ethical committee of Shahid Beheshti University of Medical Sciences, Tehran, Iran (SBMU.REC.2015.529, 1/4/2015). The present study was conducted at the Physiotherapy Research Center, Shahid Beheshti University of Medical Sciences, Tehran, Iran.

At first demographic data were collecting and then a digital camera (Sony, DSC WX80, Japan, 2013) was used to measure head and neck postures in standing position. The camera was placed at distance of $1.5 \mathrm{~m}$ from the participant on a fixed base. The height of camera was adjusted to the level of the participant's shoulder (14). The participant was instructed to stand in a natural standing position she felt comfortable and distribute body weight evenly between feet, maintaining that position throughout the assessment (3). Self-Balanced position was used to standardize head and neck posture of the participants. The participant moved the head and neck into full extension and flexion and reduced the range of movement gradually to stop movement and keep the neck and head in habitual posture (14). For measuring CVA, C7 spinous process and tragus of the ear were marked. Three pictures from lateral view of each sides of the participants were taken. Images were transferred to computer and Image J software was used to measure the CVA.

The CVA was measured as an angle between a horizontal line passing $\mathrm{C} 7$ spinous process and a line connecting the $\mathrm{C} 7$ to the tragus of the ear (14). If the average CVA calculated from the two sides was less than $48^{\circ}$, the participants were considered to have FHP (14). Since many of the previous studies considered CVA less than $48-50^{\circ}$ as FHP, in 
the current study authors considered CVAs less than $48^{\circ}$ as FHP (14).

After evaluation of CVA, maximal isometric force of CC flexion was measured and then ultrasonography of LCo was done in resting and contraction states $(50 \%$ of maximal isometric CC flexion).

\section{Evaluation of maximal isometric force of CC flexion}

A custom-made device was used to measure maximal isometric force of CC flexion (figure 1). Prior to the main study, a reliability study was performed to assess the reliability of this device and the results showed that this device was reliable for measuring isometric force of CC flexion. The device was calibrated before use. This device has three horizontal and two vertical bars. The level of horizontal bars was adjustable. The vertical bars were fixed to the wall and floor. Two load cells were mounted on the first horizontal bar and were connected to the monitors. The second and the third horizontal bars had wide, adjustable, and rigid plates to fix thorax and pelvis of participants during isometric force test-

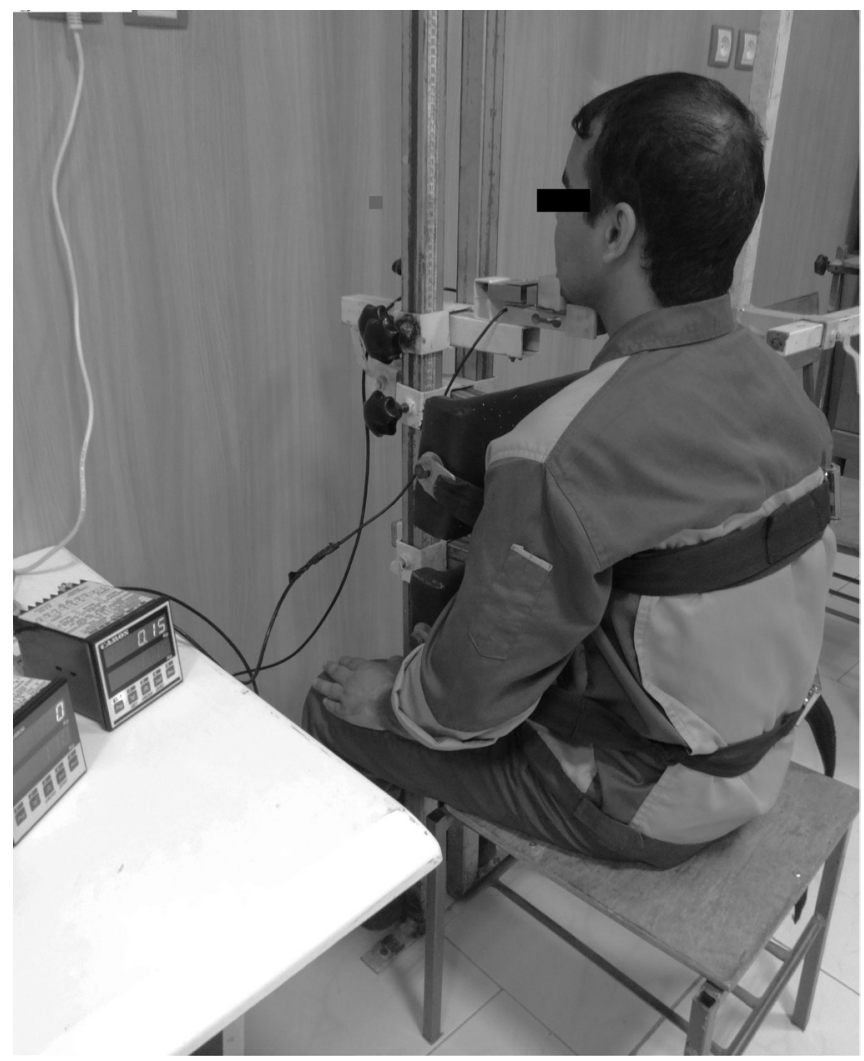

Figure 1. Position of participants during measurement of isometric force of craniocervical flexion. ing. Superior and inferior load cells (ZEMIC, H3-C3 and ZEMIC, L6D-C3, respectively) were placed in front of and under the mandible for measurement of isometric force of $\mathrm{CC}$ extension and flexion, respectively. For measurement of isometric force of CC flexion, participants were asked to sit on a stool facing the device, hips, and knees at $90^{\circ}$ of flexion, feet on the floor, and both hands on the thighs. The participants were required to relax muscles of the shoulders, arms, and legs (15) and keep this position during isometric force testing. They were also asked to focus on a point on the facing wall, at eye level during the test, to minimize flexion of the neck during testing. Participants performed a nodding movement of the head on the load cell for evaluation of isometric force of CC flexion. They were instructed to keep jaws closed during CC flexion and push on the inferior load cell as hard as they could. The command of push was given by a loud, recorded voice and mandibular depression was controlled during CC flexion.

Prior to the main test, participants were instructed about the correct form of CC flexion and performed three submaximal isometric CC flexion (8). After one-minute rest, participants performed three maximal isometric CC flexion. Each maximal isometric CC flexion lasted 5 seconds with 45 seconds rest (16). If the result of the third maximal isometric CC flexion represented improvement greater than $10 \%$, more tests were done until the improvement remained under $10 \%$. The best value of maximal isometric CC flexion was used for data analysis.

\section{Ultrasonography of LCo in resting state}

Ultrasonography images of LCo muscle were taken using an ultrasonography device (Ultrasonic scanner, HS 2100, Honda Electronic Co., Japan) with a $7 \mathrm{~cm}$ linear probe in B-mode $(7.5 \mathrm{MHz})$. The participants were asked to sit in the relaxed state on a chair, keep knees and hips at $90^{\circ}$ of flexion, and maintain head and neck in the neutral position. Participants were asked to maintain upper arms in the resting position by sides, forearms, and hands on the thigh. Because any change in position would change muscle thickness, the position of participants was checked during ultrasonography.

\section{Ultrasonography of longus colli muscle in contraction state}

After obtaining image of LCo in resting state, image of this muscle was obtained in contraction state $50 \%$ of maximal isometric CC flexion). For this purpose, participants were requested to do submaximal isometric CC flexion until monitor demonstrated $50 \%$ of maximal isometric force of 
CC flexion. An assistant helped examiner to capture image of LCo muscle. In this study we measured thickness of LCo muscle in $50 \%$ of maximal isometric contraction of CC flexion because, previous studies indicated that there was not significant changes in muscle thickness in contraction levels above $50 \%$ of maximum isometric contractions (17).

It should be noted that to visualize LCo muscle using ultrasonography in resting and contraction state, the probe was placed transversely at the C6 level. Three images of LCo muscle were frozen and stored for future analysis. To obtain each image (resting state, contraction state), the probe was removed and repositioned at the same level. All participants were right handed and images of the LCo muscle were taken on right side (figures 2,3). The thickness of the LCo muscle was measured using ultrasonography on each image. The average thickness obtained from three ultrasound images was used for the statistical analysis.

In this study, authors measured thickness of LCo muscle in resting and contraction state and thickness change of this muscle between resting state and contraction state. Muscle thickness in resting and contraction state was normalized to

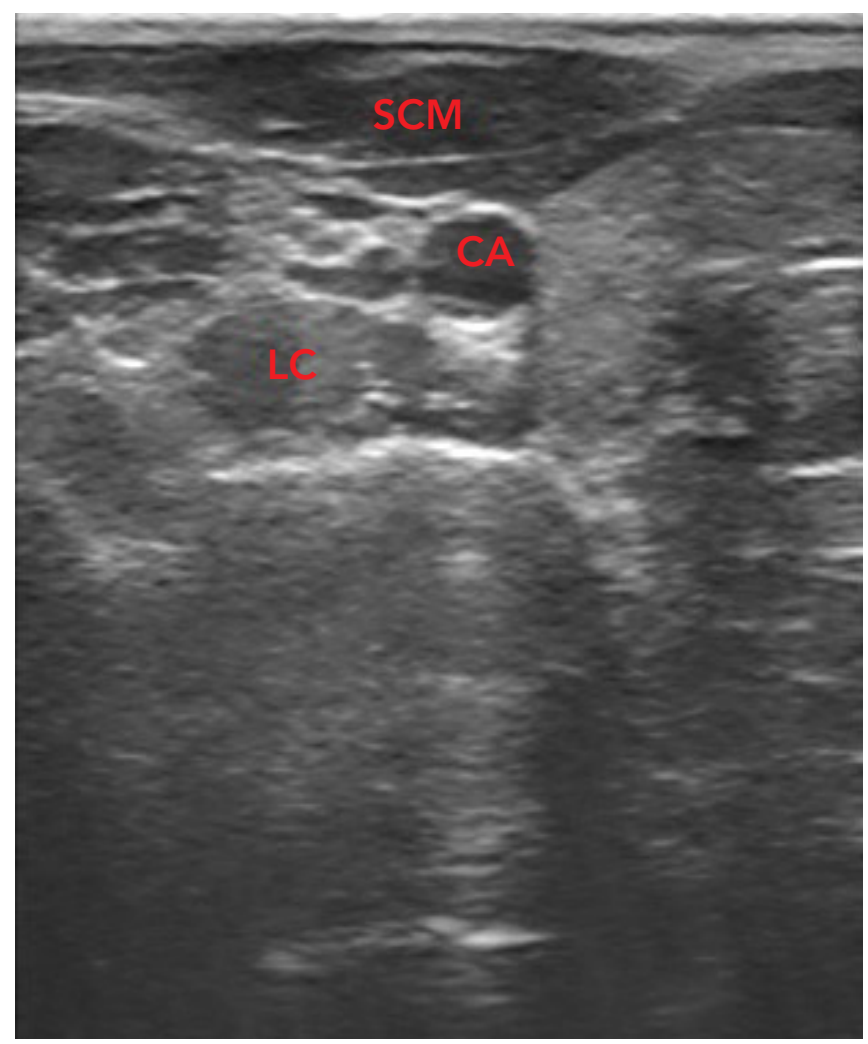

Figure 2. Ultrasonography image of longus colli muscle in resting state (LCo: longus colli, SCM: sternocleidomastoid, CA: carotid artery). body weight and thickness change was measured by difference of thickness between resting and contraction state.

\section{STATISTICAL ANALYSIS}

Data were analyzed using "SPSS Inc. Released 2007. SPSS for Windows, Version 16.0. Chicago, SPSS Inc.". The normality of the variables was assessed by Shapiro-Wilk test. The results showed normal distribution for thickness and thickness change of LCo muscle. Thickness of LCo muscles at resting and contraction states and thickness change of this muscle between resting and contraction state were compared between two groups using Independent-Samples $\mathrm{T}$ test. The correlation between thickness change of LCo and CVA was determined using Pearson's correlation coefficient. The level of significance was $\mathrm{p}<0.05$.

\section{RESULTS}

Table I represent demographic data and CVA of the participants. Mean and standard deviation of LCo muscle thick-

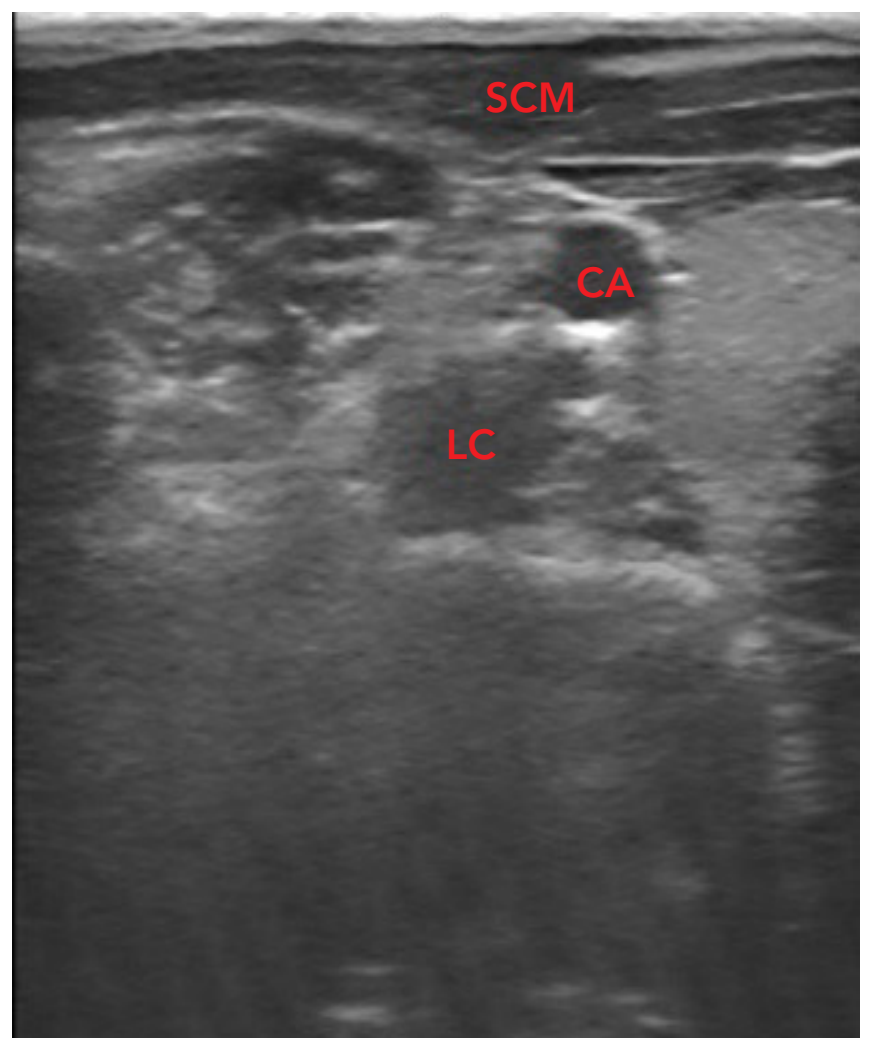

Figure 3. Ultrasonography image of longus colli muscle in contraction state (LCo: longus colli, SCM: sternocleidomastoid, CA: carotid artery). 
ness (resting state, contraction state) and thickness change of this muscle between resting and contraction state has been shown in table II. Correlation between thickness change of LCo and CVA is demonstrated in table III.

The results of the present study demonstrated that although LCo muscle thickness at resting and contraction state were greater in control group than in FHP group but, these differences were not significant $(\mathrm{p}>0.05)$. Greater thickness change of LCo muscle between resting and contraction state was observed in the control group compared with FHP group and this difference was significant ( $\mathrm{p}$ :
0.02). There was no significant correlation between thickness change of LCo and CVA.

\section{DISCUSSION}

Due to the fact that FHP is one of the most prevalent work-related poor postures and musculoskeletal disorders (18), in the presented study the authors made an attempt to clarify whether there was significant difference in regard of performance of LCo muscle between women with and without FHP. To the best of the authors' knowledge, there

Table I. The mean (standard deviation) of demographic data and craniovertebral angle in women with and without FHP ( $\mathrm{n}=$ 35 in each group).

\begin{tabular}{|c|c|c|c|}
\hline Variable & Group & Mean (SD) & P Value \\
\hline \multirow{2}{*}{ Age (year) } & Control & $25.18(5.52)$ & \multirow{2}{*}{0.89} \\
\hline & FHP & $24.94(5.13)$ & \\
\hline \multirow{2}{*}{ Height (m) } & Control & $1.63(0.04)$ & \multirow{2}{*}{0.87} \\
\hline & FHP & $1.63(0.06)$ & \\
\hline \multirow{2}{*}{ Weight (kg) } & Control & $60.72(10.09)$ & \multirow{2}{*}{0.81} \\
\hline & FHP & $60.17(9.35)$ & \\
\hline \multirow{2}{*}{ BMI $\left(\mathrm{kg} / \mathrm{m}^{2}\right)$} & Control & $22.81(3.7)$ & \multirow{2}{*}{0.81} \\
\hline & FHP & $22.6(3.59)$ & \\
\hline \multirow{2}{*}{ CVA (degree) } & Control & $54.26^{\circ}\left(1.88^{\circ}\right)$ & \multirow{2}{*}{$<0.001$} \\
\hline & FHP & $43.76^{\circ}\left(1.55^{\circ}\right)$ & \\
\hline
\end{tabular}

FHP: forward head posture, BMI: body mass index, CVA: craniovertebral angle.

Table II. The mean (standard deviation) of thickness and thickness change of longus colli muscle in women with and without $\mathrm{FHP}(\mathrm{n}=35$ in each group) in $\mathrm{mm}$.

\begin{tabular}{|c|c|c|c|}
\hline Variable & Group & mean $(\mathrm{SD})$ & P Value \\
\hline \multirow{2}{*}{ Thickness of LCo in resting state } & Control & $12.82(2.46)$ & \multirow{2}{*}{0.8} \\
\hline & FHP & $12.67(2.75)$ & \\
\hline \multirow{2}{*}{ Thickness of LCo in contraction state } & Control & $15.82(2.8)$ & \multirow{2}{*}{0.17} \\
\hline & FHP & $14.89(2.86)$ & \\
\hline \multirow{2}{*}{ Thickness change of LCo } & Control & $2.99(1.76)$ & \multirow{2}{*}{0.02} \\
\hline & FHP & $2.22(0.81)$ & \\
\hline
\end{tabular}

LCo: longus colli, FHP: forward head posture, SD: standard deviation.

Table III. Correlation between thickness change of LCo and CVA.

\begin{tabular}{|c|c|c|c|}
\hline \multirow[b]{2}{*}{ Variable } & \multicolumn{3}{|c|}{ Pearson Correlation coefficient } \\
\hline & Group & & P Value \\
\hline \multirow{2}{*}{$\begin{array}{l}\text { Correlation between thickness change of } \\
\text { LCo and CVA }\end{array}$} & Control & -0.02 & 0.9 \\
\hline & FHP & 0.26 & 0.12 \\
\hline
\end{tabular}

LCo: longus colli, CVA: craniovertebral angle, FHP: forward head posture. 
are limited studies conducted on muscle performance in FHP so, the authors cannot compare the results of present study with the results of previous studies. In previous studies the researchers did not evaluate thickness change of LCo muscle during CC flexion. Regarding this point that muscle thickness change can be informative about muscle behavior and function (19), evaluation of this parameter in FHP is highlighted.

In the current study, thickness of LCo muscle in resting state, contraction state $50 \%$ of maximal isometric contraction of CC flexion) and thickness change of this muscle between resting and contraction state were measured and compared between women with and without FHP. The results of the presented study showed that there was only significant difference in regard of thickness change of LCo muscle between resting and contraction state between two groups. This finding can represent evidence of altered contraction pattern of LCo in FHP. Altered pattern of muscle activity of LCo may change imposed loads on cervical spine and lead to neck pain.

In the present study authors measured thickness of LCo muscle, because this muscle is an important muscle in adjusting and maintaining posture of cervical spine and controlling cervical lordosis (6) and evaluation of muscle thickness is an appropriate index of muscle activity (2).

The results of current study showed that thickness of LCo in resting state in FHP group was smaller than control group but, this difference was not significant. This result is somewhat consistent with the findings reported by Ishida et al. Ishida et al. measured association between CVA and thickness of deep neck flexor (DNF) muscles in healthy men. They reported that men with smaller CVAs had smaller DNF muscle thickness (6).

Smaller thickness of LCo in resting state in FHP group could be attributed to this point that our participants had moderate degree of FHP and FHP might not lead to structural changes in these participants. This finding can also be attributed to the participants' lack of pain, because pain is one factor that can lead to muscle atrophy (18). Smaller thickness of LCo in FHP group may also be the result of disuse of this muscles in daily living with the head translated forward in sitting position (6) or dominancy of sternocleidomastoid (SCM) muscle in neck flexion movements.

Thickness of LCo muscle in contraction state was greater in control group than FHP group but, this difference was not significant. This finding is somewhat in agreement with the results of previous studies reported decreased activity of DNF muscles in FHP (20). In FHP, LCo muscle is in mechanical disadvantage and mechanical disadvantage decrease ability of this muscle for CC flexion (21). Lower activity of muscle can lead to altered muscle size and func- tion (22). Previous studies conducted on patients with neck pain reported smaller cross-sectional area (CSA) of LCo (22), altered motor control strategy including reduced activity of DNF muscles and increased activity of superficial neck flexor muscles (SCM) during CC flexion (23). Increased activity of superficial neck flexor muscles may be a compensatory mechanism for reduced activity of DNF muscles (24). In this study we used $50 \%$ of maximum isometric contraction of CC flexion because, according to the results of previous studies there was not significant changes in muscle thickness in contraction levels above $50 \%$ of maximum isometric contractions (17).

In the present study in addition to measuring muscle thickness, we also measured muscle thickness change. Muscle thickness change during CC flexion has been neglected in previous studies. This parameter is important because muscle thickness change can be informative about muscle contraction pattern (24) and more muscle thickness changes express more muscle activity (11). Measurement of muscle thickness change of cervical muscles can indicate stabilizing role of these muscle, because deep neck muscles are more active in contraction state relative to resting state to provide stability of the cervical spine (19).

An interesting observation in the presented study was that in spite of insignificant difference of LCo muscle thickness between two groups, thickness change of this muscle between resting and contraction state was significantly different between two groups. Thickness change of this muscle was smaller in FHP group than control group. This significant difference highlighted impairment of performance of LCo in FHP. FHP alter length and length-tension relationship of the neck muscles and can lead to decreased force production ability and weakness of LCo muscle (18, 24). Previous studies indicated that FHP is associated with weakness of DNF muscles in patients with cervicogenic headache (8).

Jun et al. measured thicknesses change of the DNF and SCM muscles in subjects with and without neck pain during CC flexion. The results of this study showed that during CC flexion, as pressure increased, control group recruited the DNF more than the pain group, while the pain group recruited the SCM more (25). In another study Hussain et al. investigated differences in thickness and contraction ratio of LCo muscle between patients with neck pain and healthy subjects. The findings indicated that patients with neck pain had smaller thickness and contraction ratio than healthy subjects (19). These studies measured thicknesses change of cervical muscles in neck pain but, to the best of the authors' knowledge there was lack of similar studies in FHP to compare the results of current study with them. 
In other study Moghadam et al. investigated CSA of the DNF muscles in supine position at rest and during five stages of CC flexion in 18 students with FHP and control group. The results of their study showed no significant difference between performances of DNF muscles during CC flexion in FHP and control group (18). The current study has some differences with Moghadam et al.'s study. They measured CSA at rest and during contraction while we measured muscle thickness change. In addition, position of ultrasonography in Moghadam et al.'s study was supine and in the current study, authors used sitting position. The results of present study are not consistent with the results of Moghadam et al.'s study. This difference can be attributed to the different parameter used for comparison between groups.

Kim et al. in another study compared the immediate effects of CC flexion exercise and suboccipital release combined with CC flexion exercise on CVA, cervical flexion and extension range of motion, and muscle activities of SCM, anterior scalene, and splenius capitis during CC flexion in subjects with FHP. The results demonstrated that range of cervical flexion and extension and CVA were significantly greater after suboccipital release combined with CC flexion exercise in comparison with CC flexion exercise alone. Muscle activities of SCM, anterior scalene, and splenius capitis were significantly lower during suboccipital release combined with CC flexion exercise than during CC flexion exercise alone across all CC flexion exercise phases except the first (20).

An interesting finding in this study was significant difference of thickness change of LCo muscle between two groups even though the participants in the study reported no current neck pain. This means that alteration of muscle performance may appear sooner than structural changes. according to the results of the current study, it seems that low-load exercises emphasizing motor control is more appropriate than strengthening exercises for management of FHP.

To the best of the authors' knowledge, this study is the first study evaluated thickness change of LCo during CC flexion in sitting position in women with and without FHP. The results of current study indicated that impaired pattern of activity of LCo can lead to impaired performance of LCo in subjects with FHP. According to this finding, motor control

\section{REFERENCES}

1. Ruivo RM, Pezarat-Correia P, Carita AI. Intrarater and interrater reliability of photographic measurement of upper-body standing posture of adolescents. J Manipulative Physiol Ther 2015;38(1):74-80.

2. Goodarzi F, Karimi N, Rahnama L, Khodakarim L. Differences in cervical extensor muscles thickness on subjects with exercise (low load) may be better for management of FHP compared with strengthening exercises (high load). The current study has a number of limitations that should be taken into account when interpreting the results. Our study was conducted only on asymptomatic women with moderate degree of FHP; so, the results cannot be generalized to symptomatic women and women with severe degree of FHP. In addition, only female participants were included in the present study, therefore the results may be different in men with FHP. Similar studies could be designed to evaluate performance of LCo muscle in different degree of FHP to measure relationship between performance of LCo muscle and degree of FHP. Further studies need to be conducted on evaluation performance of LCo muscle in subjects (men and women) with severe degree of FHP to better understand muscle performance in FHP. Authors also suggest measuring performance of LCo muscle in symptomatic patients with FHP (tension type headache, cervicogenic headache, neck pain) to evaluate relationship between performance of LCo muscle and pain intensity. Current study was a cross sectional study, and it is not clear whether impaired performance of LCo is the cause or the result of FHP. In addition, we were not aware of duration of FHP to evaluate the effect of duration of FHP on performance of LCo.

\section{CONCLUSIONS}

In conclusion, the results of the present study indicated that individuals with FHP demonstrated different pattern of thickness changes of LCo muscle during CC flexion compared with control group. This type of difference can indicate different pattern of muscle activity or altered motor strategy. Thickness change of LCo muscle can be used as a valuable measurement to compare performance of this muscle between subjects with and without FHP and this parameter may reveal muscle dysfunction better than muscle thickness in resting or contraction state. The study was conducted according to the journal's guidelines (26).

\section{CONFLICT OF INTERESTS}

The authors declare that they have no conflict of interests. normal head posture and forward head posture: an ultrasonography study. JRSR 2015;2(2):23-6.

3. Silva AG, Punt TD, Sharples P, Vilas-Boas JP, Johnson MI. Head posture and neck pain of chronic nontraumatic origin: a comparison between patients and pain-free persons. Arch Phys Med Rehabil 2009;90(4):669-74. 
4. Raine S, Twomey LT. Head and shoulder posture variations in 160 asymptomatic women and men. Arch Phys Med Rehabil 1997;78(11):1215-23.

5. Shenoy S, Sodhi J, Sandhu JS. Effectiveness of strengthening exercises in the management of forward head posture among computer professionals. Indian J Physiother Occup Ther 2010;4(3):37-41.

6. Ishida H, Suehiro T, Kurozumi C, Ono K, Ando S, Watanabe $\mathrm{S}$. Correlation between neck slope angle and deep cervical flexor muscle thickness in healthy participants. J Bodyw Mov Ther 2015;19(4):717-21.

7. Moon H-J, Goo B-O, Kwon H-Y, Jang J-H. The effects of eye coordination during deep cervical flexor training on the thickness of the cervical flexors. J Phys Ther Sci 2015;27(12):3799-801.

8. Watson DH, Trott PH. Cervical headache: an investigation of natural head posture and upper cervical flexor muscle performance. Cephalalgia 1993;13(4):272-84.

9. Javanshir K, Amiri M, Mohseni-Bandpei MA, Rezasoltani A, Fernández-de-las-Peñas C. Ultrasonography of the cervical muscles: a critical review of the literature. J Manipulative Physiol Ther 2010;33(8):630-7.

10. Rahnama L, Rezasoltani A, Khalkhali-Zavieh M, Rahnama B, Noori-Kochi F. Reliability of new software in measuring cervical multifidus diameters and shoulder muscle strength in a synchronized way; an ultrasonographic study. Braz J Phys Ther 2015;19(4):279-85.

11. Karimi N, Rezasoltani A, Rahnama L, Noori-Kochi F, Jaberzadeh S. Ultrasonographic analysis of dorsal neck muscles thickness changes induced by isometric contraction of shoulder muscles: a comparison between patients with chronic neck pain and healthy controls. Man Ther 2016;22:174-8.

12. English C, Fisher L, Thoirs K. Reliability of real-time ultrasound for measuring skeletal muscle size in human limbs in vivo: a systematic review. Clini Rehabil 2012;26(10):934-44.

13. Afhami N, Sahebazamani M, Amir-Sifaddini M, GHahremani-Tabrizi K. Deep and superficial cervical flexor muscles strength in female students with forward head posture compared to normal group using electromyography and pressure bio-feedback device. J. Kerman Univ. Medical Sci 2012;18(1):70-8.

14. Salahzadeh Z, Maroufi N, Ahmadi A, et al. Assessment of forward head posture in females: observational and photogrammetry methods. J Back Musculoskelet Rehabil 2014;27(2):131-9.
15. Rezasoltani A, Ahmadi A, Jafarigol A, Vihko V. The reliability of measuring neck muscle strength with a neck muscle force measurement device. J Phys Ther Sci 2003;15(1):7-12.

16. Ylinen J, Salo P, Nykänen M, Kautiainen H, Häkkinen A. Decreased isometric neck strength in women with chronic neck pain and the repeatability of neck strength measurements. Arch Phys Med Rehabil 2004;85(8):1303-8.

17. Lee J-P, Wang C-L, Shau Y-W, Wang S-F. Measurement of cervical multifidus contraction pattern with ultrasound imaging. J Electromyogr Kinesiol 2009;19(3):391-7.

18. Moghadam RE, Rahnama L, Karimi N, Amiri M, Rahnama M. An ultrasonographic investigation of deep neck flexor muscles cross-sectional area in forward and normal head posture. J Bodyw Mov Ther 2018;22(3):643-7.

19. Hussain RI, Henry LJ, Ramli A, Othman SN, Mohan V, Mohamad HF. Investigation of the morphology of longus colli among patients with neck pain using ultrasonography a preliminary study. Bangladesh J Med Sci 2013;12(4):419-26.

20. Kim B-B, Lee J-H, Jeong H-J, Cynn H-S. Effects of suboccipital release with craniocervical flexion exercise on craniocervical alignment and extrinsic cervical muscle activity in subjects with forward head posture. J Electromyogr Kinesiol 2016;30:31-7.

21. Grimmer K, Trott P. The association between cervical excursion angles and cervical short flexor muscle endurance. Aust J Physiother 1998;44(3):201-7.

22. Javanshir K, Rezasoltani A, Mohseni-Bandpei MA, Amiri M, Ortega-Santiago R, Fernández-de-las-Peñas C. Ultrasound assessment of bilateral longus colli muscles in subjects with chronic bilateral neck pain. Am J Phys Med Rehabil 2011;90(4):293-301.

23. Jull GA, O'leary SP, Falla DL. Clinical assessment of the deep cervical flexor muscles: the craniocervical flexion test. JMPT 2008;31(7):525-33.

24. Lee H-J, Song J-M. Deep Neck Flexor and Sternocleidomastoid Muscle Thickness Change in Persons with No Current Neck Pain using Rehabilitative Ultrasonograpic Imaging. J Korean Phys Ther 2016;28(6):349-54.

25. Jun I, Kim K. A comparison of the deep cervical flexor muscle thicknesses in subjects with and without neck pain during craniocervical flexion exercises. J Phys Ther Sci 2013;25(11):1373-5.

26. Padulo J, Oliva F, Frizziero A, Maffulli N. Basic principles and recommendations in clinical and field Science Research: 2018 update. MLTJ 2018;8(3):305-7. 